\title{
TOE ROCK STABILITY FOR RUBBLE MOUND BREAKWATERS
}

\author{
Stephan Baart ${ }^{1}$ and Reinder Ebbens ${ }^{2}$ and Julia Nammuni-Krohn ${ }^{3}$ and Henk Jan Verhagen ${ }^{4}$ \\ Present design tools, as found in the Rock Manual or Coastal Engineering Manual, for the determination of toe rock \\ size for rubble mound breakwaters are based on test data with a large spread: data is relatively dispersed around the \\ centre and descriptive equations have limited applicability ranges. New research has been undertaken to contribute to \\ a more accurate description of toe rock stability. Flume tests have lead to an empirical design criterion for toe bunds in \\ very shallow water based on the Hudson-type stability number. Herein the foreshore slope turns out to have an \\ important influence. An approach with theoretical background has been used for toe bunds in surging wave \\ conditions. The resulting stability description, based on local flow velocities, has been verified with existing data sets. \\ Additional flume tests were performed to measure flow velocities at the toe bund. Results are used to calibrate the \\ velocity-approach, providing an improved design criterion.
}

Keywords: rubble mound breakwater; toe structure; toe bund; rock stability; wave load; foreshore slope.

\section{INTRODUCTION}

The Rock Manual (CIRIA et al., 2007) provides guidance for the design of rubble mound breakwater toes. This research concerns stability of rocks in 'standard size' toe bunds of conventional rubble mound breakwaters. This means trapezoidal shaped rubble bunds near the bed at the seaward extend of breakwaters. A toe bund has a negligible influence on the hydraulic performance of the breakwater, distinguishing it from a berm.

For determination of the required rock size in toe bunds, the existing formulas are those by GERDING 1993 (1) and VAN DER MEER 1998 (2):

$$
\begin{aligned}
& \frac{H_{s}}{\Delta D_{n 50}}=\left(0.24\left(\frac{h_{t}}{D_{n 50}}\right)+1.6\right) \cdot N_{o d}^{0.15} \\
& \frac{H_{s}}{\Delta D_{n 50}}=\left(6.2\left(\frac{h_{t}}{h_{m}}\right)^{2.7}+2\right) \cdot N_{o d}^{0.15}
\end{aligned}
$$

These formulas have limited applicability and limited theoretical background. Some recent developments in research on stability of rocks in toe bunds include:

- New flume tests for very shallow water and with different foreshore slopes.

- Theoretical approach to toe rock stability, verified for depth-limited surging waves.

- Additional flume tests for flow velocity near toe structures.

\section{TOE BUNDS IN VERY SHALLOW WATER}

Considerable damage of the toe structure was experienced during the commercial testing of a design that was derived in accordance with equation (2). The specific test conditions during which this unexpected behaviour occurred were noted to be shallow water and steep foreshore slopes. Additional flume research tests carried out thereafter indicate that the foreshore slope in particular seems to have considerable influence on stone stability.

\section{Experiment set-up}

The 2D physical experiments were carried out in the wave flume of BAM Infraconsult, the Netherlands. The test flume dimensions were a length of $25 \mathrm{~m}$, a width of $0.60 \mathrm{~m}$ and a height of $1 \mathrm{~m}$. The tests comprised of irregular wave fields based on a Jonswap spectrum. Reflecting waves were automatically compensated by a system provided in the wave generating paddle. Wave data were captured at the wave paddle and near the structure.

\footnotetext{
${ }^{1}$ Lievense Consulting Engineers, Breda, The Netherlands; sbaart@lievense.com

2 DHV, Amersfoort, The Netherlands; reinder.ebbens@dhv.com

${ }^{3}$ Halcrow, London, United Kingdom; NammuniJl@halcrow.com

${ }^{4}$ Delft University of Technology, Delft, The Netherlands; H.J.Verhagen@tudelft.nl
} 
Figure 1 shows the toe cross section tested, with an Xbloc ${ }^{5}$ armour unit with $D$ of $40 \mathrm{~mm}$ and mass of $49 \mathrm{~g}$. The first underlayer comprised a $W_{50}$ of $5.0 \mathrm{~g}$ and a $D_{n 50}$ of $12.4 \mathrm{~mm}$ with a grading $\left(D_{85} / D_{15}\right)$ of 1.29. The core material comprised a $D_{n 50}$ of $11.1 \mathrm{~mm}(3.6 \mathrm{~g})$ and a grading of 1.5 . This is a standard stone grading achieved by quarries. The size of the toe material to be tested was determined with the current Van der Meer toe equation (2). Stone sizes were determined for $70 \%, 80 \%$ and $100 \%$ of the design wave height $\left(H_{s}\right)$ which lead to nominal stone diameters of $1.88 \mathrm{~cm}, 2.15 \mathrm{~cm}$ and $2.68 \mathrm{~cm}$ respectively. The grading was kept relatively narrow with a maximum value of 1.5.

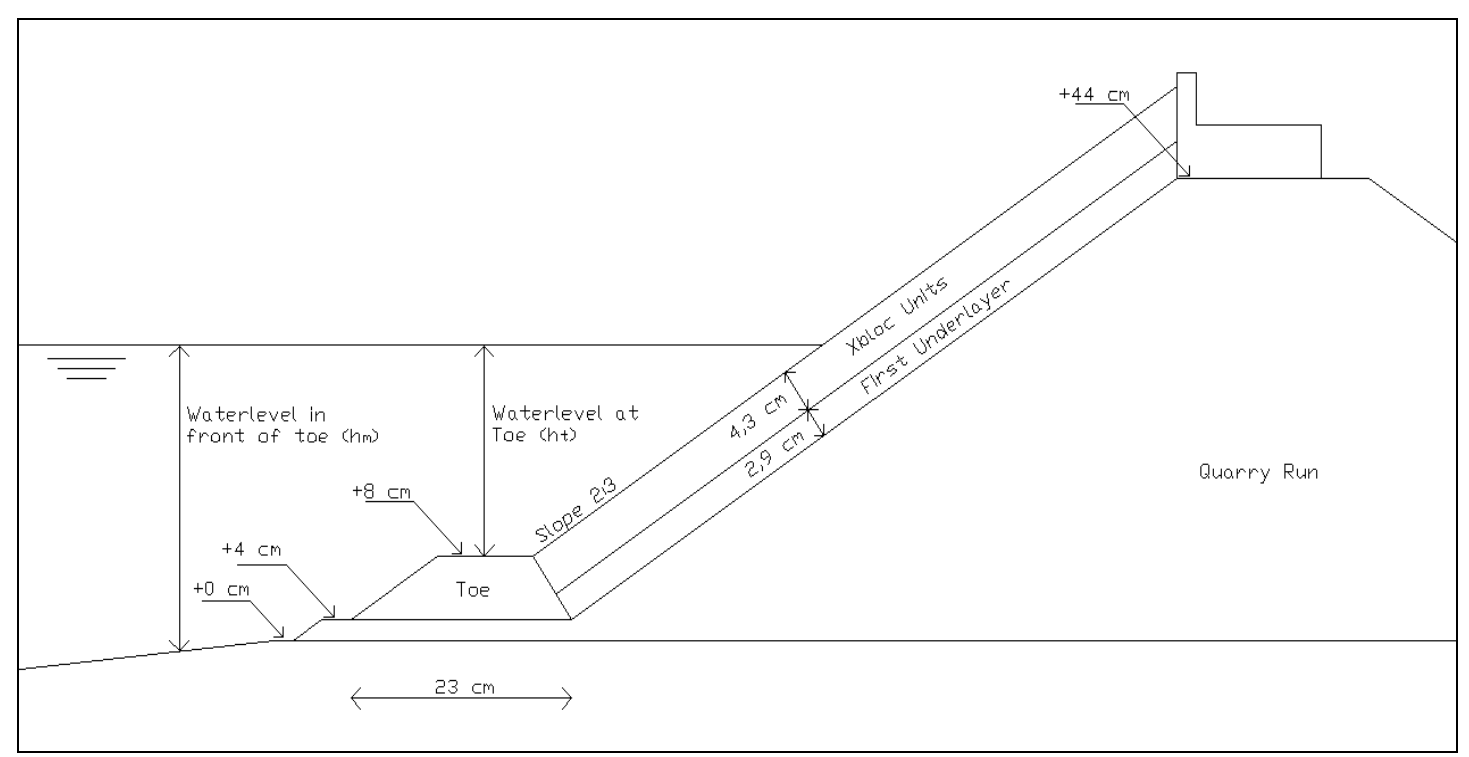

Figure 1. Cross section scale model tests.

The test program was built up in such way that the toe was tested for stability with an increasing load with respect to foreshore slope and wave height. The storm duration was set to 1000 waves, which is representative for a storm with three hours duration. Cumulative damage was observed as the toe was not rebuilt for each test. Tests were undertaken for a number of combinations of water level, wave height, wave steepness and foreshore slope:

\begin{tabular}{|l|l|}
\hline \multicolumn{2}{|l|}{ Table 1. Overview tested parameters } \\
\hline Parameter: & Value: \\
\hline$h_{t}$ & $-0.00,0.02,0.04,0.06,0.08,0.12,0.17 \mathrm{~m}$ \\
\hline$H_{s}$ & $0.06,0.08,0.10 \& 0.12 \mathrm{~m}$ \\
\hline$S_{o p}$ & $0.02 \mathrm{~m} / \mathrm{m}, 0.04 \mathrm{~m} / \mathrm{m}$ \\
\hline $\tan \left(\alpha_{\text {shore }}\right)$ & $1: 50,1: 20,1: 10$ \\
\hline
\end{tabular}

All data obtained from the experiment are presented in figure 2. The figure shows a clear difference between the three foreshore slopes. Therefore this figure confirms the expectation that foreshore slope influences toe stability. The 1:50 slope caused the smallest amount of damage. Damage development increased for steeper slopes and was highest for a 1:10 slope. From figure 2 it is also concluded that in very shallow water $\left(h_{t} / h_{m}<0.4\right)$ the influence of relative water depth, namely $h_{t} / h_{m}$, is small. The trends of the three different data clouds are about vertical for very shallow water. The effect of a more exposed toe is most likely compensated by the fact that most of the waves are already broken.

\footnotetext{
${ }^{5}$ Xbloc is a registered trademark of Delta Marine Consultants, the Netherlands
} 


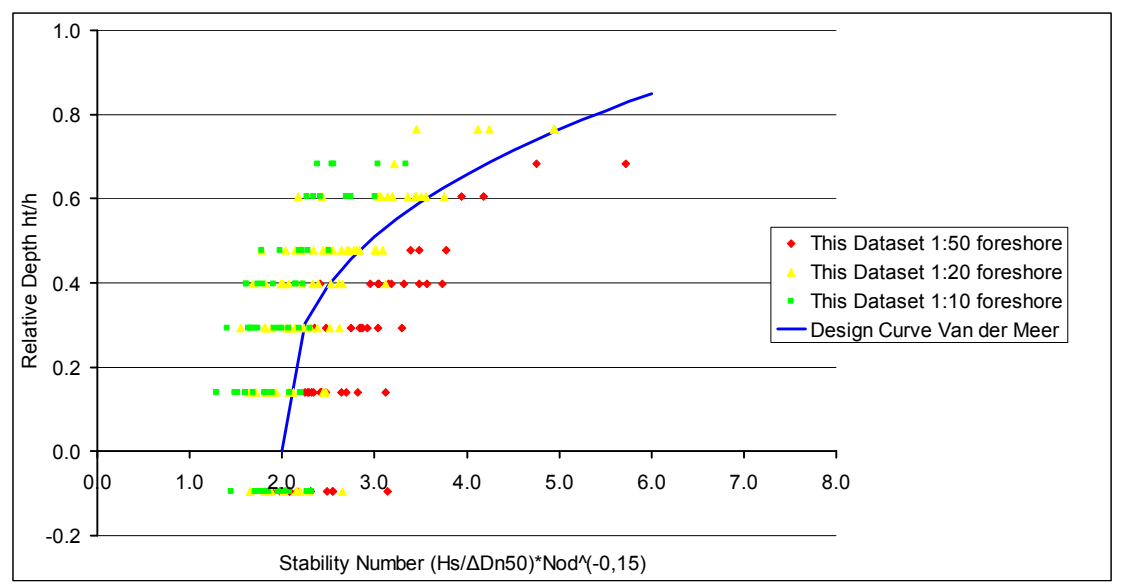

Figure 2. Results scale model tests.

\section{Analysis}

The observations, analysis and results are closely described in EBBENS 2009. A general equation for the description of the stone stability was determined considering that damage can be described as a function of wave load, strength and geometry.

$$
\text { Damage }=f\left(\frac{\text { WaveLoad }}{\text { Strength }}, \text { Geometry }\right)
$$

The parameters, describing these factors were related based on the results and observations from the scale model tests and existing empirical relationships. The Hudson-type stability number was used in the analysis because it is well known and a frequently used empirical relationship between wave load and strength. New considered geometrical parameters in these experiments were wave steepness and foreshore slope.

The analysis is done with the tests for which applies that $h_{t} / h_{m}<0.4$. Examples of found relations between individual variables are presented in figure 3 .

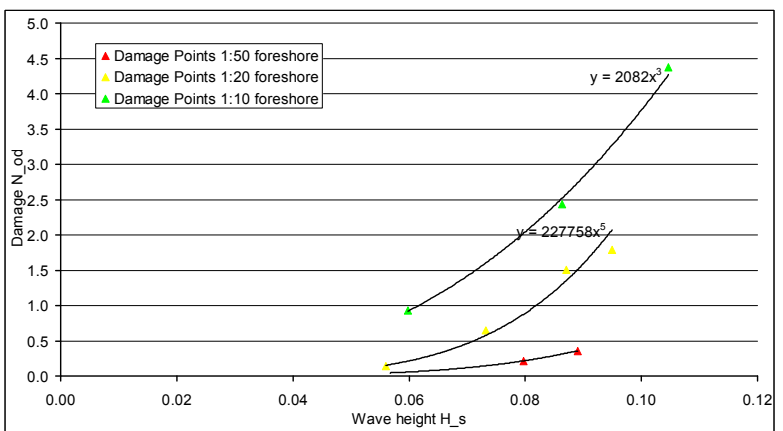

A.Wave height to damage, $S_{0}=0.02, h_{t}=0.033 \mathrm{~m}$

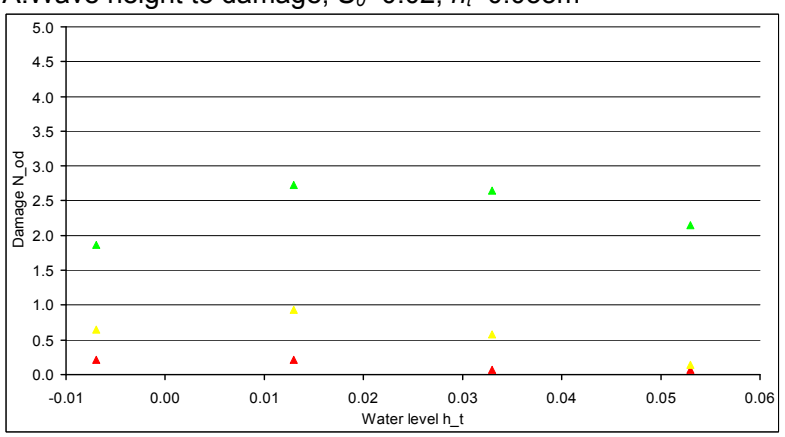

C.Water level to damage, $S_{0}=0.04, H_{s}=0.10 \mathrm{~m}$

Figure 3. Observed damage according to tested variables.

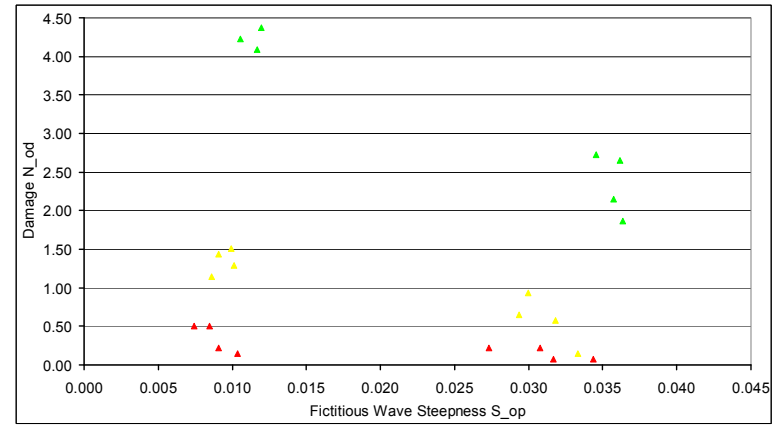

B. Wave steepness to damage, all $h_{t}, H_{s}=0.10 \mathrm{~m}$

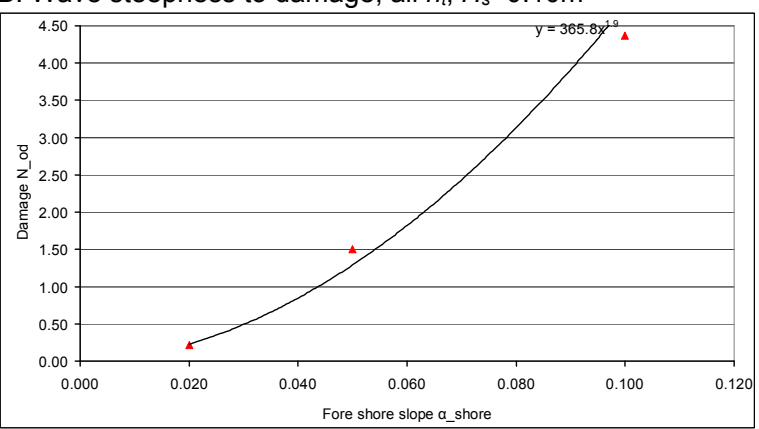

D.Foreshore slope to damage, $S_{0}=0.02, H_{s}=0.10 \mathrm{~m}, h_{t}=0.03 \mathrm{~m}$ 
Figure 3, part B indicates that wave steepness has an effect on the damage. However, given the limitation of the testing and relatively low number of data points, no trend line could be determined. Hence, the authors chose to represent the effect of the wave steepness in the damage equation by including the Iribarren number.

The Hudson stability number, with strength parameters $D_{n 50}$ and $\Delta$, and the Iribarren number are combined to describe damage:

$$
\begin{aligned}
& N_{o d}=B \cdot\left(H_{s} / \Delta D_{n 50}\right)^{4} \\
& N_{o d}=B \cdot \alpha_{\text {shore }}{ }^{2} \\
& N_{o d}=B \cdot\left(\frac{\tan \left(\alpha_{\text {shore }}\right)}{\sqrt{s_{o p}}}\right)^{2}=B \cdot \xi_{o p}{ }^{2}
\end{aligned}
$$

The assumed relationship between $H_{s}$ and $D_{n 50}$ and $\Delta$ as in equation (4) could not be proven with this dataset. Therefore the analysis was initially undertaken for a single stone diameter to reduce the number of variables and associated uncertainties when trying to establish patterns.

The results are presented in figure 4 , which excludes data for tests with $h_{t} / h_{m}>0.4$. This is done because figure 2 suggests that the water depth has an effect on stability for configurations beyond this limit. Tests results with these conditions should therefore be considered separately.

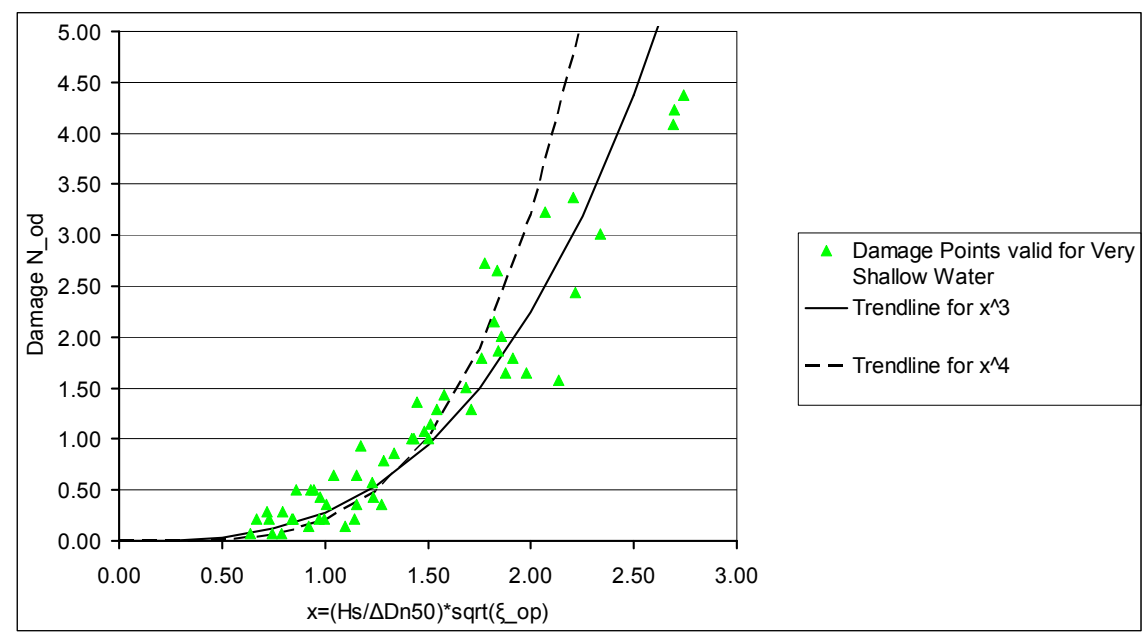

Figure 4. Curve fit for damage based on $D_{n 50}=2.15 \mathrm{~cm} \& h_{t} / h_{m}<0.4$.

The best trend line derived for the data cloud considering a continuous fit indicates a power relationship with a scaling exponent of three. The data indicates relatively large dispersion from the fit for damage values of $N_{o d}<0.5$, however for larger damage values, the data appears to be well represented by the fit.

\section{Damage description for design purposes}

Gerding and Van der Meer used the damage number $N_{o d}$ to quantify damage. $N_{o d}$ is a good damage parameter for describing the relationship between the amount of observed damage in a scale model test and the test conditions. The value represents the number of displaced rocks in a strip as wide as the nominal stone size of toe rock.

$$
N_{o d}=\frac{N}{B / D_{n 50}}
$$

$N$ is the number of displaced stones and $B$ the total width of the wave flume.

However, the acceptable value for damage $N_{o d}$ may vary on a case by case basis dependent on the design. A single value of the damage number $N_{o d}$ can imply a different damage severity per design case. The design value for damage $N_{o d}$ would depend on the size of the toe, size of the stone diameter and acceptance of damage. Therefore in this analysis, a new damage number is introduced which 
presents damage as a percentage of the total volume. This number, $N_{\%}$, provides a better interpretation of acceptable damage. $N_{\%}$ is used to derive the final design formulation.

$$
N_{\%}=100 \cdot N \cdot \frac{D_{n 50}{ }^{3}}{(1-n) \cdot V_{t o t}}
$$

$N$ is the number of displaced rock, $n$ is the porosity and $V_{t o t}$ is the volume of toe rock in the wave flume.

\section{Design equation}

The final design equation is based on the total set of model tests performed. All three stone sizes tested were considered and hence more variation is visible in Figure 5. This figure also excludes data for tests with $h_{t} / h_{m}>0.4$, similar to figure 4 .

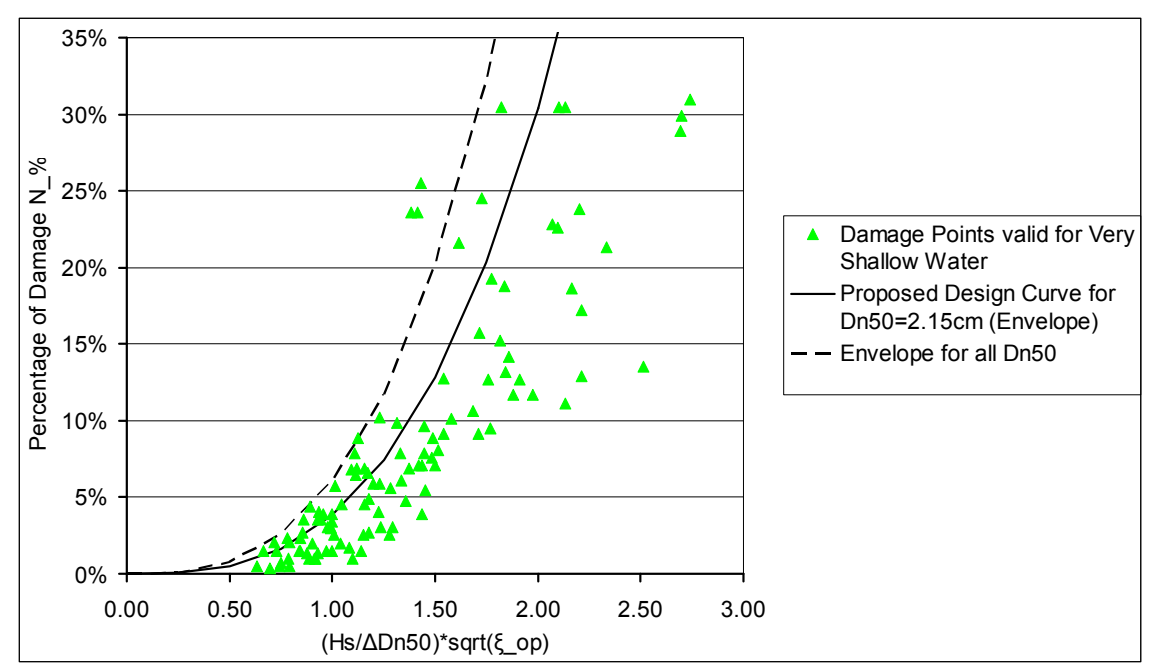

Figure 5. Design curve for toe stability in very shallow water for all stone sizes for $h_{t} / h_{m}<0.4$.

The continuous line in Figure 5 is a proposed design curve fitted to the data. The dispersion of data points from the line indicates a level of associated uncertainty and risk to the design. An estimate of the uncertainty is made by counting the number of data points on each side of the proposed design curve whilst taking the distance from each data point into account. An acceptable level of uncertainty for design is deemed to be $10 \%$ uncertainty on the risky side. This means that $90 \%$ of the results are expected to fall within the fitted design curve value and to be "safe".

In addition, an envelope is provided that encompasses all data points until $N_{\%}=10 \%$ which is considered the conservative design equation. The resultant equations are presented in equation (9) and (10).

The conservative design equation (dashed line) is:

$$
N_{\%}=0.06 *\left(\frac{H_{s}}{\Delta D_{n 50}} \sqrt{\xi_{o p}}\right)^{3}
$$

The equation for the proposed design curve (continuous line) with $10 \%$ uncertainty is:

$$
N_{\%}=0.038 *\left(\frac{H_{s}}{\Delta D_{n 50}} \sqrt{\xi_{o p}}\right)^{3}
$$

Equation (11) is the proposed design equation (10) for toe stability in very shallow water, but now rewritten in the format of typical design guidance such as the Rock Manual (CIRIA 2007):

$$
\frac{H_{s}}{\Delta D_{n 50}}=3.0 \cdot \frac{N_{\%}^{1 / 3}}{\sqrt{\xi_{o p}}}
$$


The Iribarren number is given by:

$$
\xi_{o p}=\frac{\tan \left(\alpha_{\text {foreshore }}\right)}{\sqrt{H_{s} / L_{o p}}}
$$

The Iribarren number is based on slope angle of the foreshore, the near shore significant wave height and the deep water wave length. The final accompanying diagram for the design equations is given in figure 6 .

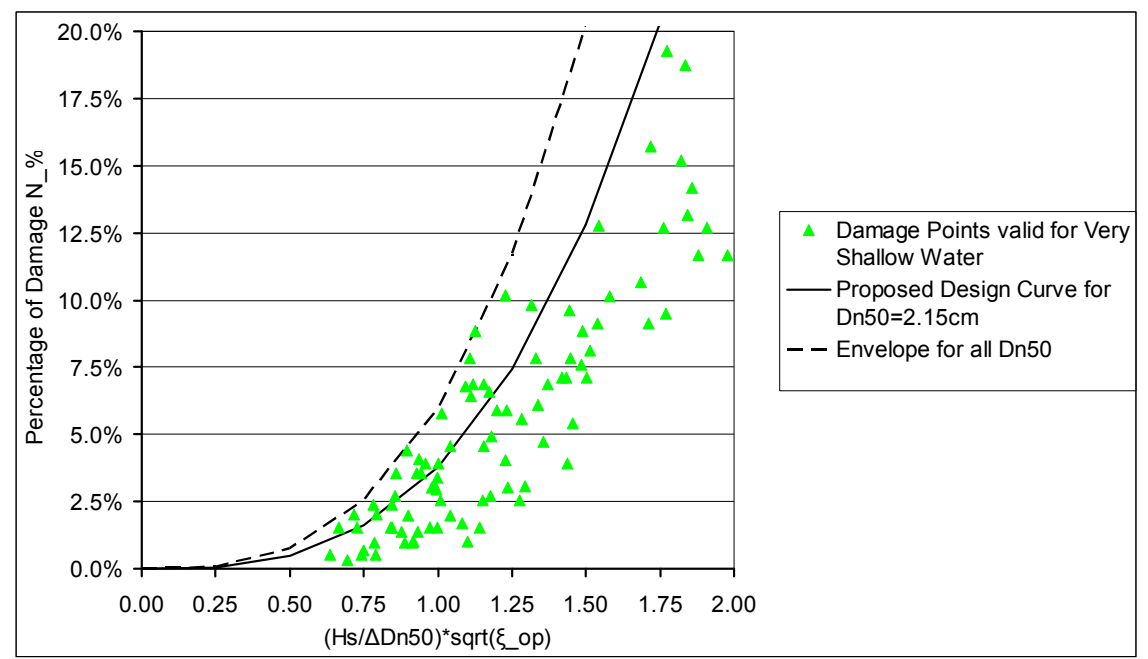

Figure 6. Proposed design equation for toe stability in very shallow water for $h_{m} / H_{s}<2.0$.

Note that this figure includes test results for which applies $h_{m} / H_{s}<2.0$ (here the limiting criterion is not given by $h_{t} / h_{m}$ ). The criterion of figure 6 is the inverse of the breaker index. These tests are selected to only take into account one hydro-dynamical situation. This is the situation where an exposed toe is attacked by breaking waves.

During the curve fit procedures which result in figures 5 and 6 , more attention was paid to the main area of interest regarding design. This means the area with acceptable damage levels. During the experiment, a difference in damage development was observed between swell waves and wind waves. The swell waves caused more damage, probably due to the longer wave period. Hence, the design conditions for swell waves are proposed stricter. The design values for very shallow water are given in terms of $N_{\%}$ :

- $\quad$ For swell waves $N_{\%}=5 \%$ is advised.

- $\quad$ For wind waves $N_{\%}=10 \%$ is advised.

A range of validity is given for the design equation. The table below gives the range of parameters tested for which the proposed design equation is considered valid.

\begin{tabular}{|c|c|c|}
\hline Parameter: & Symbol: & Range: \\
\hline Damage level & $N_{\%}$ & $<30 \%$ \\
\hline Fore shore angle & $\tan \left(\alpha_{\text {shore }}\right)$ & $1: 50-1: 10$ \\
\hline Fictitious wave steepness & $s_{o p}$ & $0.008-0.04$ \\
\hline Iribarren number using $T_{p}$ & $\xi_{o p}$ & $0.3-0.9$ \\
\hline Relative water depth in front of toe & $h_{m} / H_{s}$ & $<2.0$ \\
\hline Stability number & $H_{s} / \Delta D_{n 50}$ & $1.5-3.5$ \\
\hline Toe material gradation & $D_{85} / D_{15}$ & $<2.0$ \\
\hline
\end{tabular}




\section{TOE ROCK STABILITY ASSESSMENT BASED ON LOCAL VELOCITIES}

\section{Limitations of previous approaches}

The Van der Meer equation (2) is a curve fit to the data set of GERDING 1993. The spread of Gerding's data points around Van der Meer fitted curve increases with increasing relative toe depth. For example for $h_{t} / h_{m} \approx 0.7$, the stability number is approximately between 2.6 and 6.1 , which results in a difference in rock weight class by a factor ten. Therefore an improved parameterisation for toe rock stability is desirable to optimise design and reduce costs.

The Gerding and Van der Meer formulas use the same relationship between $N_{o d}$ and stability, namely a power relationship with an exponent of 0.15 , see equations (1) and (2). There is no theoretical basis for this relationship. Further, the empirical basis noted in Gerding's report is not very evident. Nonetheless, this power relationship has a large influence on the scatter, as is shown by analysis in BAART 2008. Another disadvantage of the power relationship is that valuable test results with $N_{o d}=0$ cannot be used in Van der Meer's curve fit, because this would result division by zero in the vertical axis of figure 2 .

The forces on rocks in deeper toe structures are different from the forces acting on the primary armour material. The stability problem for these cases appears to be more similar to the stability of nearbed structures. Then the rock properties $\Delta D_{n 50}$ may be proportional to $u^{2}$, where $u$ is the local flow velocity. The velocity $u$ is influenced by the wave height $H$, but also by the wave period $T$ (or wave length). Therefore the use of $H_{s} / \Delta D_{n 50}$ may be less appropriate for rocks in deeper toes.

\section{Approach based on local physical process}

A new approach to this problem, as described in BAART 2008, is used in an attempt to find a relationship that predicts toe rock stability more accurately. This method considers the theoretical background in order to determine other more suitable (dimensionless) parameters for the description of toe rock stability. In general, it is argued that the local velocity should be an important parameter. This velocity can be derived using existing theoretical equations. Furthermore it would be interesting to base the stability description on local flow velocities, because a clear trend is that numerical volumeof-fluid models are more and more accurately describing local flow velocities at coastal structures. Combining these analytical and numerical techniques is a future possibility.

The approach of this flow velocity method is to regard the toe rock stability as an equilibrium problem on local scale at the toe bund. This implies to estimate the local conditions for the toe rocks, by assessing stabilizing and destabilizing mechanisms as load and resistance. General wave conditions such as water depth and wave height and period are used to estimate local flow conditions that act as forces on a rock.

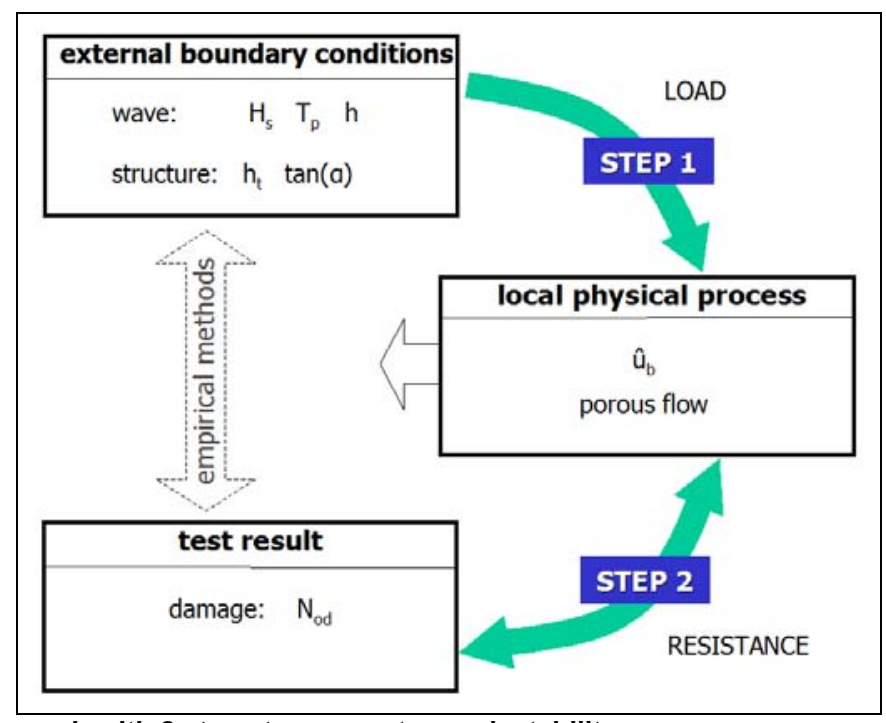

Figure 7. Velocity approach with 2 steps to assess toe rock stability. 
Former methods have attempted to empirically couple the test set-up conditions to the damage result directly. The new method can be regarded as a 2-step approach (see figure 7):

- First the local flow velocity at the toe bund is assessed using the test set-up (boundary conditions),

- Secondly is reviewed what the critical local flow conditions are for the rocks in the test.

The presentation of the results in a diagram is chosen as follows. The horizontal axis shows the ratio of load and resistance, which can be regarded as the stability number or rather the dimensionless relative load on a rock. On the vertical axis is the test result, i.e. the amount of damage expressed in $N_{o d}$. By displaying the damage result on the vertical axis separately, it is not required to assume a (power-) relationship between the general test conditions and damage result. Besides avoiding unnecessary scatter, this presentation makes it easier for a designer to interpret the sensitivity range of the damage to expect for certain design conditions. The following figure 8 shows the result that is aimed for with this method:

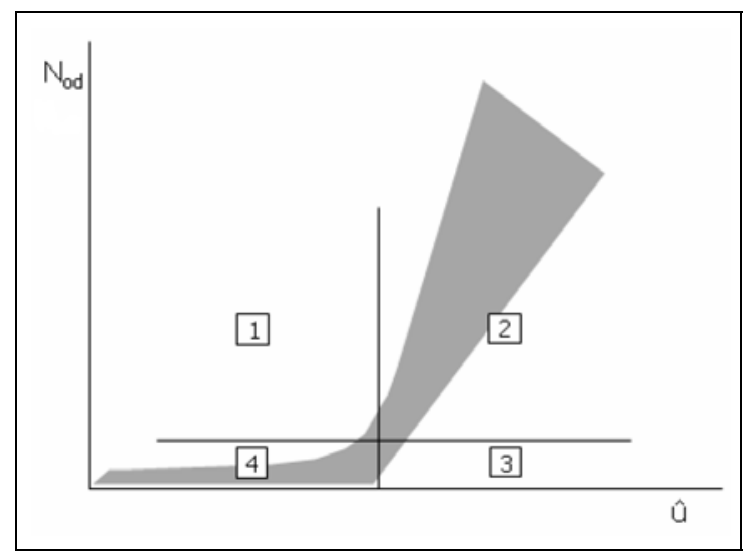

Figure 8. Schematic presentation of desired result of rock stability assessment.

The vertical line could be interpreted as a "threshold of movement" or critical value of relative load. The resemblance between this parameter model (set of mathematical equations) and the physical model tests is relevant if no test results are present in quadrant 1 and 3 of the figure: Having no test results in quadrant 1 ensures stability in breakwater design and no test results in quadrant 3 prevents overdimensioning. A relationship for damage development beyond the critical load (in quadrant 2) is considered not particularly relevant for design practice and therefore it is not aimed to fit a curve through this data cloud.

\section{APPROACH OF LOCAL VELOCITY APPLIED TO TOE BUNDS IN SURGING WAVES}

The velocity approach is based on the hypothesis that rock movement commences when the destabilising forces (load) on the rocks become larger than the stabilising forces (resistance). The load is expressed in amplitude of local flow velocity at the toe bund $\left(\hat{u}_{b}\right)$ and the resistance in the critical value of this velocity $\left(\hat{u}_{b c}\right)$.

This means that rocks will move if $\hat{u}_{b} / \hat{u}_{b c}>1$. With this method the stability of toe rocks was assessed considering surging waves on the front slope of a permeable breakwater, as for example in Gerding's tests. In this assessment:

- $\hat{u}_{b}$ is the summation of the incoming wave and the reflected wave rushing down the slope;

- $\hat{u}_{b c}$ is calculated with a stability criterion from literature, which is adapted for the effect of porous outflow through the rubble mound structure.

Various methods exist for the calculation of these parameters. The theoretically developed methods are typically based on regular waves. Calibration of the equations to account for irregular waves was undertaken with test data of GERDING 1993 and DOCTERS VAN LEEUWEN 1996. The best results, in terms of absolute and relative resemblance between parameter model and physical tests, were obtained with the equations as described in the following sections. 


\section{Occurring local flow velocity}

Local flow velocity was calculated as the sinusoidal addition of incoming wave and down rush. The theoretical approach indicates that for regular waves both contributions have the same wave period. For the incoming wave linear wave theory was used. Down rush was calculated as flow velocity due to average conversion of potential energy to kinetic energy. Run up and run down were calculated with criteria from the Rock Manual (CIRIA 2007), see BAART 2008. The following equations were applied:

$$
\hat{u}_{b}=\sqrt{\left(\hat{u}_{b i} \cdot \sin \left(\varphi_{T A}\right)\right)^{2}+\left(\hat{u}_{b i} \cdot \cos \left(\varphi_{T A}\right)+\hat{u}_{b d r}\right)^{2}}
$$

in which

$$
a_{b d r}=\gamma_{d r} \sqrt{2 g\left(R_{u} / 3+R_{d} / 2\right)}
$$

and

$$
\hat{u}_{b i}=\omega \frac{H}{2} \frac{\cosh \left(k\left(h_{m}-h_{t}\right)\right)}{\sinh \left(k h_{m}\right)}
$$

and

$$
\varphi_{T A}=k x=\frac{2 \pi}{L} \cdot L_{T A}
$$

with $L_{T A}$ as in figure 9

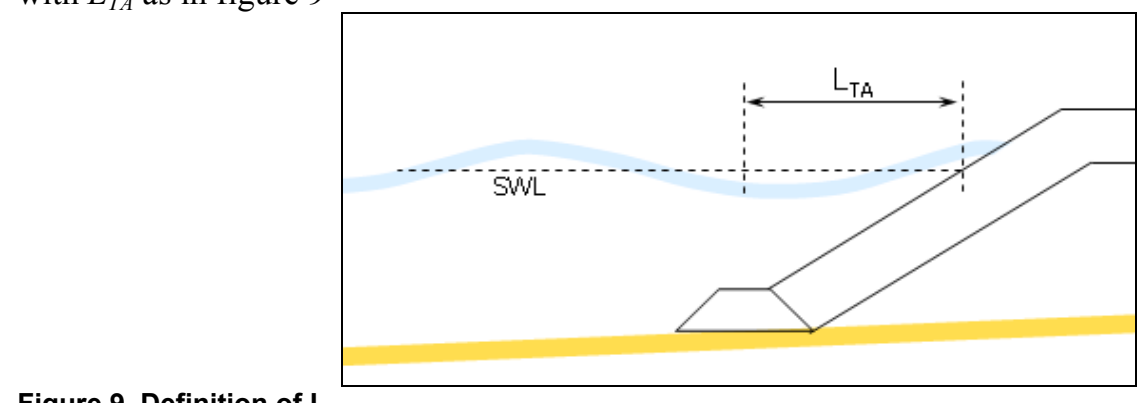

Figure 9. Definition of $L_{T A}$.

In the above analysis a regular wave with a height $H$ was assumed. For irregular waves using significant wave height $H_{s}$ provided better results than using $H_{2 \%}$. Recent research as mentioned in The Rock Manual (CIRIA 2007), recommends for wave current interaction in shallow water the use of $T_{m}$ ${ }_{1,0}$ as governing period parameter. Therefore preferably $T_{m-1,0}$ should be used for analysis, since this should be more representative for the wave period in near shore conditions. Unfortunately only $T_{p}$ was available in Gerding's tests. Therefore $T_{m-1,0}$, was estimated with $T_{m-1,0} \approx 0.9^{*} T_{p}$.

\section{Critical flow velocity}

Critical flow velocity for rocks in the toe bund is calculated with an adapted form of the criterion of RANCE et al. 1968. This criterion is set-up for bed rock stability in coastal conditions. This criterion was adapted for the porous flow through the breakwater structure that exits at the toe bund. This porous flow may be an important parameter in the equilibrium of a toe rock, since the flow pushes up the toe rocks. This can be interpreted as if the head gradient fictitiously decreases the specific weight of the rocks, which can be shown theoretically with an equilibrium of forces, see BAART 2008. The strength of the uplift is determined by the head gradient in the structure, which is estimated by:

$$
i=\frac{\Delta h}{\Delta x}=\frac{H / 2+R_{u}}{L_{T A}+R_{u} / \tan \alpha}
$$

An empirical fit constant $C_{P F}$ is introduced, since this maximum possible head gradient will not be present in the toe bund. The term $C_{P F}{ }^{*} i$ is introduced in the Rance-Warren criterion, which leads to:

$$
\left.\hat{u}_{b c}=\left(0.46 \sqrt{T} \cdot\left(\left(\Delta-C_{P F} \cdot i\right) g\right)^{1.5} \cdot D_{n 50}\right)^{(1 / 2.5}\right)
$$


With the preceding equations, the following figure 10 can be obtained for the dataset of Gerding:

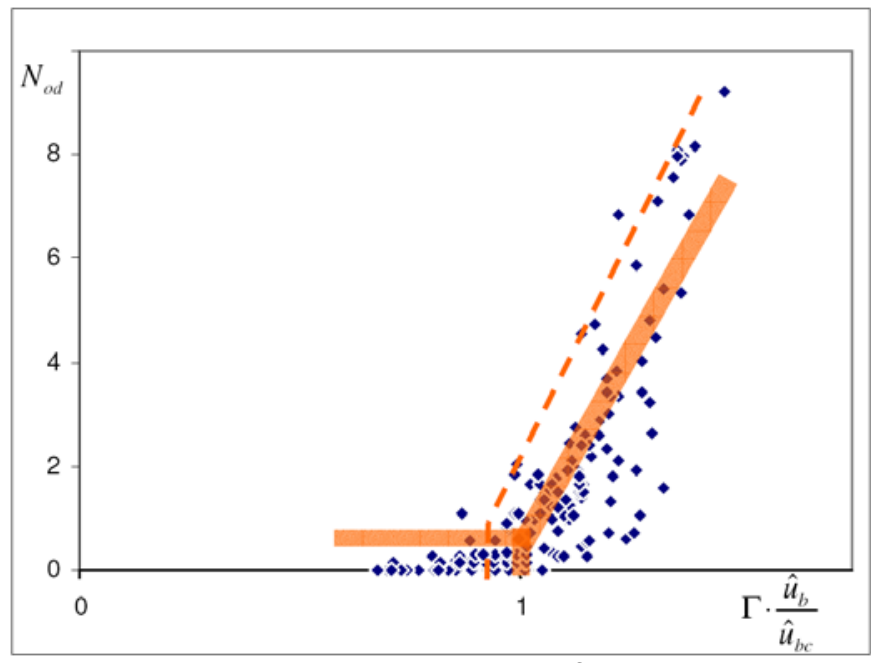

Figure 10. Analysis result with $\Gamma=1.05$ and a design line (dashed, for $\Gamma \approx 0.94$ ).

The vertical axis shows the damage number $N_{o d}$ and the horizontal axis shows the stability number of this method, namely $\hat{u}_{b} / \hat{u}_{b c}$. The parameter $\Gamma$ is a fit constant. In this figure the value of $\gamma_{d r}$ is 0.45 (equation 14) and $C_{P F}$ is 0.4 . Only the boundary conditions and the test result (amount of damage) are known for the data set of Gerding. Therefore it is not possible to calibrate the separate steps in this method. This makes it difficult to determine the correct value of each fit parameter separately. The values presented provide a reasonable result when combined in this way, but do not provide specific knowledge on the separate terms in the approach.

Figure 10 shows the fit of the velocity approach to the data set of Gerding. Therefore, the values of the fit parameters, as mentioned above, are only valid for conditions similar to the tests. The values may be used for:

- $\quad$ Permeable rubble mound breakwater with a rough front slope with $\tan \alpha \approx 0.67$

- $H_{s} / h_{t}>0.5$

- $H_{s} / h_{m}>0.35$

Due to natural irregularities of the waves and rocks, there is not a clear transition value but rather a transition range between stable and unstable toe rocks. An analysis of the datasets of Gerding and Docters van Leeuwen indicates that the transition between stable and unstable toe rocks is approximately between $0.4<N_{o d}<0.8$ (ideally to be found only in quadrant 4 in figure 8 ). Figure 10 shows that above the critical flow velocity the amount of damage to expect is not easily predictable. Therefore it is advised not to use higher values of $N_{o d}$ in breakwater design. This damage number does however not express how severe the amount of damage is to the toe bund to fulfil its function in the structure.

\section{FLOW VELOCITY AT TOE BUNDS}

Flume tests with velocity measurement

Additional flume tests were performed to measure flow velocities at the toe bund (NAMMUNI 2009). The main objective was to obtain initial results that may provide insight in the physical processes at the toe bund. This data may help lay a foundation for further development of the two-step velocity model towards a full theoretical model. The experiments were set-up to be comparable to the experiments of Gerding.

The flow velocity was measured with Acoustic Doppler Velocimeters (ADV's) at several locations above the toe bund. Tests with regular and irregular waves were performed. Phase differences were analysed with Lissajous plots and amplitudes of flow velocities were assessed with Fourier-analysis. 


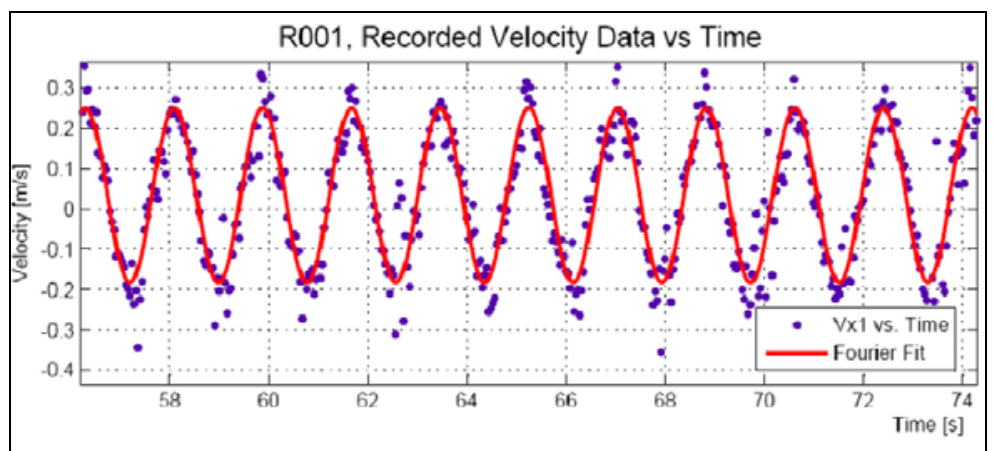

Figure 11. Example of a Fourier fit to orbital velocity measurements in regular waves.

Analysis shows that with respect to the incoming wave, a considerable additional velocity component is visible, which is likely to indicate the presence of the predicted down rush influence. A phase shift between the wave and the velocity is noticeable for high breaker indices but is not as strong or absent with low breaker indices. This difference is probably due to the relatively strength of the downrush component.

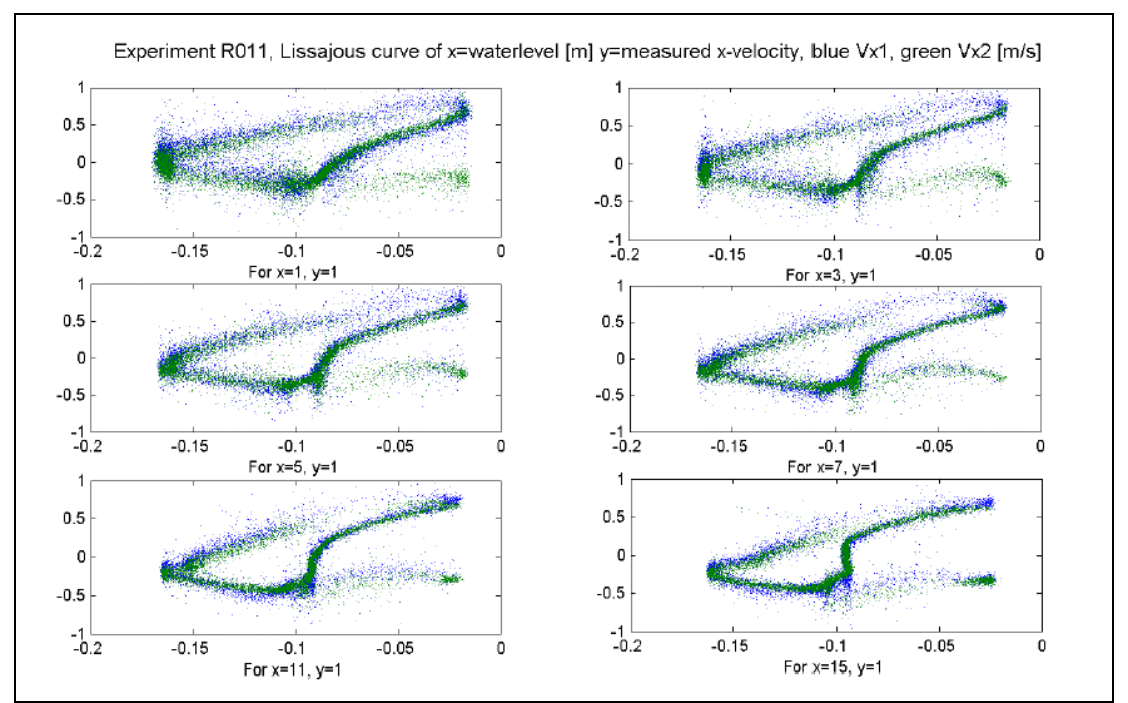

Figure 12. Example of phase shift between wave and velocity with $h_{m} / H_{s}=2.0$.

\section{Calibration of the velocity method to measured flow velocities}

An approximately mid-point position along the breakwater toe has been taken to compare calculated and measured velocity. The following figure shows a selection of the measured flow velocities versus calculated flow velocities for one velocity probe $(7 \mathrm{~cm}$ horizontal and $1 \mathrm{~cm}$ vertical from the toe):

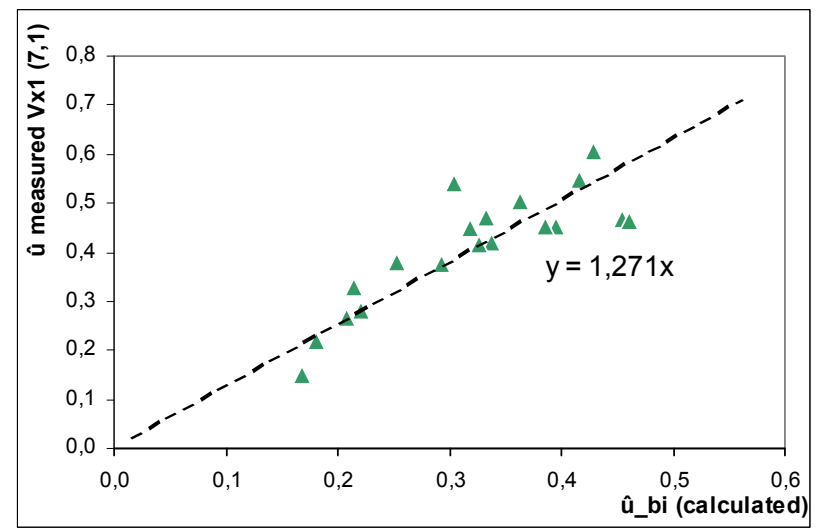

Figure 13. Comparison of calculated and measured velocity. 
The measured velocities are for regular wave tests and velocities are calculated with offshore $H_{s}$ of the experiments of NAMMUNI 2009. It is found that measured $\hat{u}_{b}$ is approximately 1.3 times calculated $\hat{u}_{b i}$. It is tentatively estimated that for irregular waves and calculation with a nearshore wave height $H_{s}$ instead of the offshore wave height the ratio will be about 1.5.

These velocity measurements show that in the original comparison in BAART 2008, flow velocities were overestimated. The combined flow velocity of incoming and reflected wave (down rush) should be roughly about 1.5 times the flow velocity of the incoming wave only.

Preliminary comparisons show reasonable results, with calibration factors for $\hat{u}_{b i}$ at $100 \%$ and for $\hat{u}_{b d r}$ of $\gamma_{d r}=0.15$ (instead of 0.45 previously). With these factors a ratio of about 1.5 on average is indeed obtained between measured $\hat{u}_{b}$ and calculated $\hat{u}_{b i}$.

This modification however reduces the absolute calculated value of occurring $\hat{u}_{b}$. This implies that also for $\hat{u}_{b c}$ a calibration factor (namely $\gamma_{b c}$ ) is required. This is because the ratio of $\hat{u}_{b} / \hat{u}_{b c}$ (horizontal position in figure 10) should remain on average the same, since in general the Gerding data fits well in the original analysis. In other words, the numerator $\hat{u}_{b}$ and denominator $\hat{u}_{b c}$ should have a similar correction. Comparison shows that $\hat{u}_{b c}$ reduced to $55 \%$ (i.e. $\gamma_{b c}=0.55$ ) yields a reasonable result, see figure $14 \mathrm{~b}$. The reduction factor for $\hat{u}_{b c}$ may be required because rocks on the seaward edge of the bund are less stable than on the bed in the experiments of RANCE et al. 1968.

\section{ADDITIONAL ANALYSIS FOR FORESHORE SLOPE IN VELOCITY METHOD}

An additional analysis is performed with the velocity method by BAART 2008 and the effect of foreshore slope as found in EBBENS 2009. The effect of difference in foreshore slope is indicatively regarded for the data sets by GERDING 1993 and DOCTERS VAN LEEUWEN 1996.

As mentioned previously, analysis by Ebbens has shown that for his new experiments $H_{s}$ (and therefore also $\hat{u}_{b i}$ ) relates to the square root of $\xi_{o p}$. It is attempted to include this effect in the velocity method to see whether it may be applicable. Since the velocity approach was calibrated to the experiments of Gerding (foreshore slope $\tan \alpha=0.05$ ), the influence of the foreshore slope on Docters van Leeuwen can be included by multiplication of $\hat{u}_{b}$ with the following factor (19):

$$
\gamma_{\text {foreshore }}=\left(\frac{\tan \alpha_{\text {foreshore }}}{0.05}\right)^{0.5}
$$

which is 0.63 for Docters van Leeuwen. The following figures show that with these additional comparisons both data sources match better.
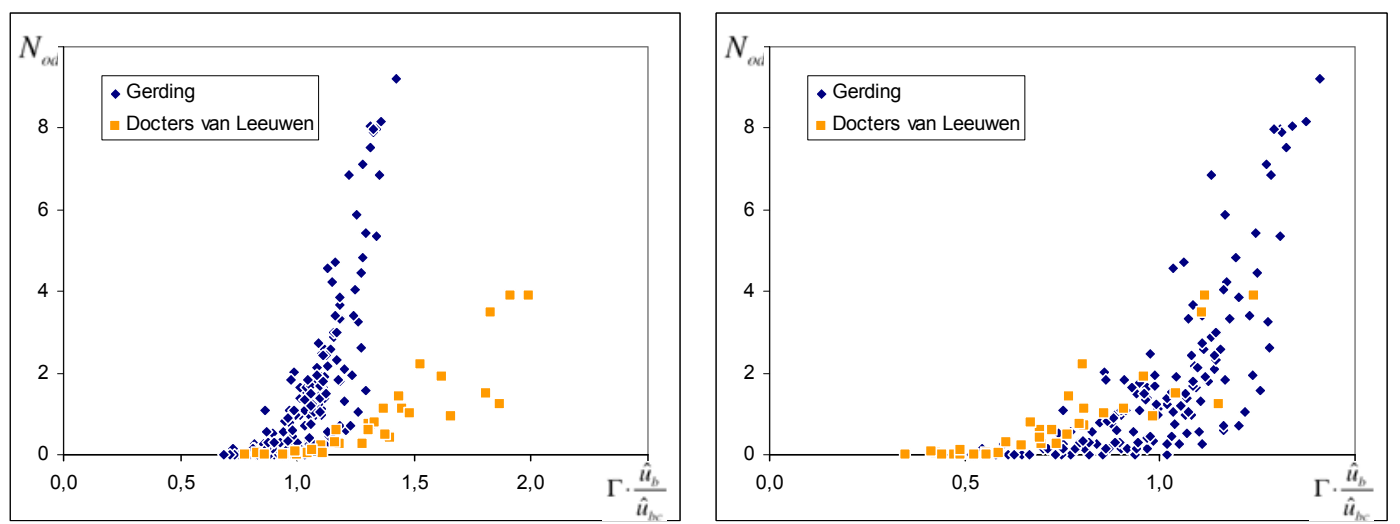

Figure 14a and 14b. Original comparison in BAART 2008 (14a) and similar figure with $\gamma_{d r}=0.15, \gamma_{b c}=0.55, \Gamma=1$ and a compensation for foreshore slope (14b).

This is a rough investigation, but still figure 14 shows that indeed the datasets match better when an influence of the foreshore slope is included. A better match between the data sets of Gerding and Docters van Leeuwen is obtainable with a slightly higher factor than 0.63 , thus with a lower exponent than 0.5 in equation 19, for example 0.35 . This may on the one hand indicate that the effect of foreshore slope is smaller for deeper toes, but on the other hand other unknown parameters may also have influence, such as shape of the rocks (not known for Gerding). 


\section{CONCLUSIONS}

The present design tools by GERDING 1993 and VAN DER MEER 1998 for the stability of rocks in toe bunds of rubble mound breakwaters lack a reasonable degree of accuracy for adequate choices in required rock size. Main causes are an inappropriate power curve between stability and the amount of damage and the limited applicability of the Hudson-type stability parameter in fully submerged conditions. New research aims to distinguish between a design method for toe bunds in very shallow water $\left(h_{t} / h_{m}<0.4\right)$ and a method for toe bunds in submerged conditions.

New flume tests show that the foreshore slope has a relevant influence on toe rock stability. For very shallow water, the effect can be described by introducing the square root of the foreshore Iribarren number. In very shallow water the relative toe depth is not an important parameter.

For deeper submerged toe bunds, a velocity-based approach with more theoretical background does improve accuracy in the description of stability of toe rocks. This was obtained by assessment of the local physical process at the toe bund. The approach is verified with existing data sets.

Additional flume tests indicate that the reflected wave (down rush) may indeed have influence on toe rock stability.

The difference in flume test results of Gerding and Docters van Leeuwen may (partly) be explained by the difference in foreshore slope.

\section{RECOMMENDATIONS}

In the theoretical approach by BAART 2008, sinusoidal addition of the incoming wave velocity and down rush is one of the most determining parameters. Theoretically this is influenced by $\tan \alpha$ of the seaward slope of the breakwater. This influence has not been calibrated, since the breakwater slope was not varied in the available test series. Furthermore the permeability has not been taken into consideration.

\section{ACKNOWLEDGMENTS}

For this research, the following institutions are greatfully acknowledged:

- Delft University of Technology

- Royal Boskalis Westminster NV (Hydronamic)

- BAM Infraconsult

\section{REFERENCES}

BAART, S.A. 2008. Toe structures for rubble mound breakwaters: analysis of toe bund design tools and a method for toe rock stability description, MSc thesis, Delft University of Technology, http://repository.tudelft.nl/

CIRIA, CUR, CETMEF. 2007. The Rock Manual: The use of rock in hydraulic engineering (2nd edition), CIRIA, London

DOCTERS VAN LEEUWEN, L. 1996. Toe stability of rubble-mound breakwaters, MSc thesis, Delft University of Technology, http://repository.tudelft.nl/

EBBENS, R.E. 2009. Toe structures of rubble mound breakwaters: stability in depth limited conditions, MSc thesis, Delft University of Technology, http://repository.tudelft.nl/

GERDING, E. 1993. Toe structure stability of rubble mound breakwaters, MSc thesis, Delft University of Technology, http://repository.tudelft.nl/

NAmmuni-KroHN, J. 2009. Flow velocity at rubble mound breakwater toes, MSc thesis, Delft University of Technology, http://repository.tudelft.nl/

RANCE, P.J. AND WARREN, N.F. (1968) Threshold of movement of coarse material in oscillatory flow, proceedings of 11th ICCE, London, p. 487-491

VAN DER MEER, J.W. 1998. Geometrical design of coastal structures, in: Dikes and revetments: Design, maintenance and safety assessment, Pilarczyk, K.W. (ed.), Balkema, Rotterdam 